\title{
Soleus motoneurone excitability in man: an indirect approach for obtaining quantitative data
}

\author{
A. J. M c C OM A S, M. M IR S K Y, F. VELHO, A N D A. STRUPPLER \\ From the Neurologische Klinik der Technischen Universität München, München, West Germany
}

S U MMARY Using combinations of $H$ and $T$ reflexes, spatial summation has been studied in the human soleus motoneurone pool. With certain assumptions, mathematical treatment of the results yielded estimates of the thresholds of motoneurones to monosynaptic activation by Ia nerve fibres. It was found that, on average, about 62 EPSPs were required to discharge a motoneurone and that a single Ia fibre supplied about 139 neurones.

Using intracellular recording the responses of motoneurones to monosynaptic inputs from primary endings of muscle spindles have been investigated in various species and in the cat in particular (see Eccles, 1957, and Discussion). These studies have enabled the excitatory postsynaptic potentials (EPSPs) evoked by single impulses in Ia nerve fibres to be measured. In addition, estimates have been made of the degree of branching, or divergence, of Ia fibres within the motoneurone pool. For obvious reasons it has not been possible to apply the same experimental techniques to man, and consequently quantitative data concerning the synaptic connections between primary afferent nerve fibres and motoneurones have been largely lacking. The purpose of this paper is to demonstrate that the problem of synaptic connectivity in man can be approached indirectly, provided certain major assumptions are permitted. A preliminary report has appeared elsewhere (McComas et al., 1979).

The method to be described makes use of the twin phenomena of subliminal fringes and spatial summation described by Sherrington (1929). The term "subliminal fringe" was applied to those cells situated around a zone of discharging neurones, which received some excitation themselves but insufficient to initiate impulses. Sherrington recognised that if two groups of afferent fibres were stimulated simultaneously, parts of the subliminal fringes could overlap and some of the cells would then gather sufficient excitation to discharge impulses. This was "spatial summation." Because of this extra fraction

Address for reprint requests: Professor A. J. McComas, Department of Medicine (Neurology), McMaster University Medical Centre, 1200 Main Street West, Hamilton, Ontario. Canada L85 4J9.

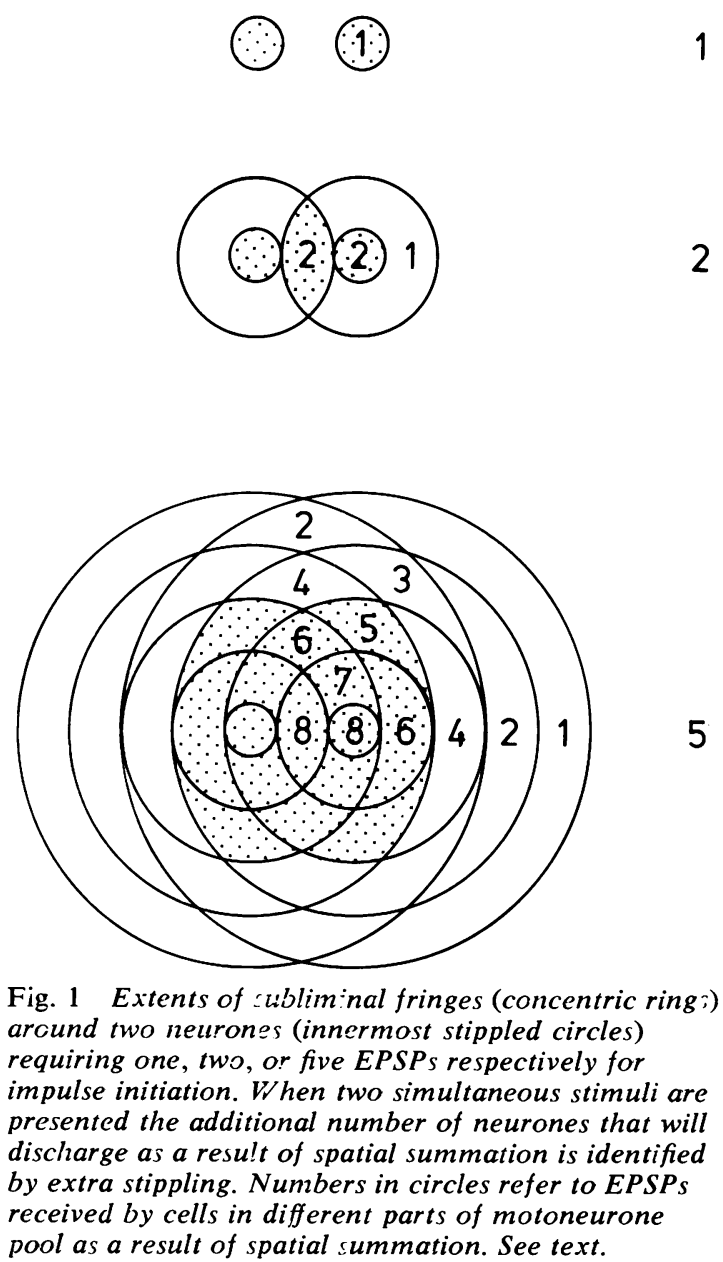
091 
of discharging cells, the response to two simultaneous stimuli would exceed the sum of the responses to the same stimuli delivered separately. It is evident that the subliminal fringe will be increased for cells with relatively high thresholds, that is, for cells requiring larger numbers of EPSPs for impulse initiation. Figure 1 shows that the opportunity for spatial summation must also be greater for these cells. This relationship would suggest that if the degree of spatial summation could be measured it might then be possible to determine thresholds of the cells, provided that the EPSPs sum in a linear manner (Burke, 1967). We will now show that, subject to certain assumptions, mathematical treatment of the situation is indeed possible.

Suppose that a single la fibre branches to make excitatory synaptic connections with $d$ cells within a pool of $M$ motoneurones. After one impulse in this fibre $d$ neurones will receive one EPSP while $M-d$ cells will remain unaffected. The chance of any one cell receiving an EPSP will be $d / M$.

If on the next occasion an additional Ia fibre is activated and also fires a single impulse, there will be $2 d$ EPSPs distributed throughout the motoneurone pool. Under these conditions the chance of any one cell gathering two EPSPs-that is, one from each of the la fibres-will be $(d / M)^{2}$. The number of neurones in this state will be $d .(d / M)$. The number of neurones receiving only one EPSP will now be $2 d-\left(2 d^{2} / M\right)=$ $2 d[1-(d / M)]$.

Similarly, if a third Ia fibre is activated, the number of cells gaining three EPSPs will be $d^{3} / M^{2}$. The number of cells with two EPSPs will be equal to the cells previously receiving two EPSPs $\left(d^{2} / M\right.$, see above), minus those cells which have acquired a third EPSP $\left(d^{2} / M \cdot d / M\right)$, plus a new population of cells, formerly receiving one EPSP, which have now gathered a second $(2 d[1-(d / M)] . d / M)$ :

$$
\frac{d^{2}}{M}+\frac{d}{M} \cdot 2 d\left(1-\frac{d}{M}\right)-\frac{d^{2}}{M} \cdot \frac{d}{M}=\frac{3 d^{2}}{M}\left(1-\frac{d}{M}\right)
$$

The number of cells having only one EPSP will be $3 d$, the total number of EPSPs delivered, minus twice the number of cells receiving two EPSPs and three times the number of cells gathering three EPSPs. That is, the total number of cells with one EPSP will be:

$$
3 d-2\left[\frac{3 d^{2}}{M}\left(1-\frac{d}{M}\right)\right]-\frac{3 d^{3}}{M^{2}}=3 d-\frac{6 d^{2}}{M}+\frac{3 d^{3}}{M^{2}}
$$

Thus, if there are 1000 cells in the motoneurone pool and three Ia fibres are activated, each of which divides to supply 100 motoneurones randomly, then there will be:

$$
\begin{gathered}
\frac{100^{3}}{1000^{2}}=\text { one cell with three EPSPs } \\
\frac{3 \times 100^{2}}{1000}\left(1-\frac{100}{1000}\right)=27 \text { cells with two EPSPs } \\
\text { and } \\
300-\frac{6 \times 100^{2}}{1000}+\frac{3 \times 100^{3}}{1000^{2}}=243 \text { cells with three EPSPs }
\end{gathered}
$$

The same approach can be used to calculate the numbers of motoneurones receiving different excitatory inputs when larger numbers of Ia fibres are activated.

The next aspect to consider is the result of applying two volleys, in different populations of Ia fibres, to the same motoneurone pool simultaneously. For simplicity, imagine that each volley involves only three Ia fibres and that, as in the example above, each fibre fires a single impulse into its 100 branches. Suppose also that the motoneurone pool comprises 1000 cells and that only three EPSPs are needed to initiate an impulse (that is, $y=3$ ).

Under these circumstances each volley, if delivered separately, would have been expected to excite one cell with three EPSPs, thereby causing it to discharge When the two volleys are given together, howevera there will be partial overlap of the two subliminat fringes. Thus, some of the cells receiving two EPSP from one volley will receive one or two EPSPs from the other volley and consequently discharge.

In the present example such an occurrence woul involve:

$$
27 \times \frac{(27+243)}{1000}=7.3 \mathrm{cells}
$$

Finally, some cells receiving one EPSP from one volley will gather two EPSPs from the other. Summation of this kind would involve:

$$
243 \times \frac{27}{1000}=6.6 \text { cells }
$$

Since only three EPSPs are needed to cause a $\mathbb{D}$ motoneurone to discharge there will now be a total of $\overrightarrow{\vec{B}}$ $2+7.3+6.6=15.9$ cells responding when the two volleys are given together.

Recalling that each volley by itself would have only fired a single motoneurone, the facilitation, $F$, produced by the two volleys together may be expressed as:

$$
\frac{15.9}{2}=8.0
$$

In summary, if 1000 motoneurones have similar thresholds (see Discussion) and if two weak excitatory volleys are presented, each of which fires a single cell by itself but which together cause 16 cells to discharge, it can be deduced that the excitatory threshold for the motoneurone is three EPSPs and that each volley involves only three Ia fibres. 


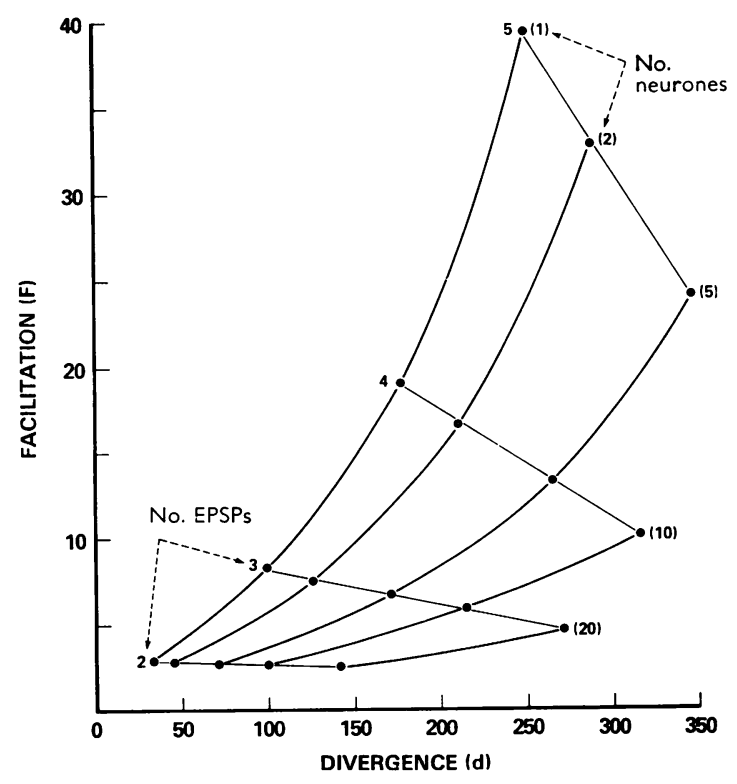

Fig. 2 Amount of facilitation ( $F$ ) to be expected for populations of motoneurones with different thresholds for impulse initiation (ie different critical numbers of EPSPs $(y))$ and four different degrees of axonal branching. The critical numbers of EPSPs are indicated by values at left of curves and are assumed to correspond to the numbers of Ia fibres activated (see text). Numbers at right in parentheses show populations of motoneurones reflexly excited by single volleys.

Figure 2 shows the facilitation to be expected when the firing thresholds of motoneurones, the degrees of branching (divergence) of Ia fibres, and the sizes of the reflex discharges (to one volley alone) are altered. For each curve it is assumed that the two impulse volleys responsible for spatial summation are of equal size. In agreement with Fig. 1 it can be seen that facilitation is greatest when the firing thresholds of the cells are raised. Facilitation is also larger when relatively small excitatory inputs are employed. Figure 2 also shows that, if the sizes of the initial reflex responses and the amount of facilitation are measured, it is possible to determine the average number of EPSPs required for impulse initiation together with the degree of axonal divergence. In plotting Fig. 2 it has been assumed that the number of EPSPs required for impulse initiation also corresponds to the number of axons stimulated. It can be shown mathematically that, since most experiments in this study involved reflexes with fewer than 10 motoneurones participating, this assumption is valid.

In the experiments to be described the impulse volleys in separate populations of Ia fibres were obtained first, by applying weak electrical stimuli to the tibial nerve behind the knee so as to elicit H reflexes (Hoffmann, 1918), and second, by lightly percussing the Achilles tendon, thereby evoking $T$ (tendon) reflexes.

The amount of facilitation, $F$, will then be equal to $H T /(H+T)$, where $H$ and $T$ are the neurones discharging in the $\mathrm{H}$ and tendon reflexes respectively, and $H T$ represents the combined response to paired electrical and mechanical stimulation.

It is well established that weak electrical stimulation of the tibial nerve preferentially activates muscle afferent fibres capable of evoking monosynaptic responses in homonymous motoneurones (Hoffmann, 1918; Magladery et al., 1951) and, by analogy with extensive studies in animals, there is little doubt that the sensory fibres involved are of the Ia moiety. Similarly, it has been shown in animals that the primary endings of the muscle spindles have the lowest thresholds to rapid stretch, such as that produced by a tendon tap (Lundberg and Winsbury, 1960; Matthews, 1972), and are connected to Ia fibres. There are no a priori grounds, however, for supposing that the two types of stimulation employed in this study would have selectively activated the same Ia fibres. Further, given the small sizes of the stimuli, it is unlikely that any of the Ia fibres would be activated by both types of stimuli by chance (see Discussion).

It should be noted that it is not actually necessary to know $M$, the population of motoneurones in the pool, to find either the number of EPSPs required to discharge a cell, $y$, or $d$, the extent of axonal divergence (branching). Provided the reflexly-discharging cells amount to $0.1,0.2,0.5,1$, or $2 \%$ respectively of the total population, the five curves in Figure 2 will be applicable to the results. Experimentally, the percentage of motor units, and hence of motoneurones, participating in an $\mathrm{H}$ or $\mathrm{T}$ reflex may be determined by comparing the amplitude of that response to the muscle $M$ wave evoked by maximal $\alpha$-motor nerve fibre stimulation. In practice it is difficult to obtain small $\mathbf{H}$ and $T$ reflexes of equal amplitude and instead the average size of the two responses can be used for comparison with the $\mathbf{M}$ wave. The resulting percentage of the motoneurone pool can then be interpolated between two of the curves in Fig. 2 for the determination of $y$ and $d$.

\section{Methods}

Experiments were conducted on 18 legs of 15 healthy young adults (four men and 11 women) free of neurological disease. All subjects volunteered for the experiments and were paid for their services. Subjects lay prone on a couch and were encouraged to relax. 
The ankle of the leg under investigation was fixed in a holder at a right angle. Ethical problems were discussed and resolved at the Neurological Clinic before the experiments started.

\section{STIMULATING AND RECORDING ARRANGEMENT}

The recording electrodes were a pair of metal discs $12 \mathrm{~mm}$ in diameter (Beckmann Ltd), coated with electrode jelly. The stigmatic electrode of the pair was placed in the midline over the soleus muscle while the reference electrode was attached to the skin of the heel. The earth (ground) electrode was a strap which was soaked in saline and fastened around the leg below the knee. The signals were fed into a Tektronix oscilloscope (type 5103) amplifier; they were also entered into an averaging device (Nicolet model 1072).

Electrical stimuli were applied to the tibial nerve in the popliteal fossa through a pair of pad electrodes, $7 \mathrm{~mm}$ in diameter, soaked in saline. The stimuli were rectangular current pulses, $0.5 \mathrm{~ms}$ in duration, delivered from a Disa Multistim.

Mechanical stimuli were delivered to the Achilles tendon by a spring loaded hammer, which enabled the force to be adjusted. The contact of the hammer with the skin triggered the averager and, when necessary, the Disa stimulator. The two types of stimuli were delivered separately or together in a random sequence until eight responses to each of the three types of stimulus had been averaged. The intervals between successive stimuli were approximately 10 seconds. When the $\mathrm{H}$ and $\mathrm{T}$ stimuli were paired, the latter was arranged to precede the former by $4.0-8.5 \mathrm{~ms}$, the selected interval being that which gave the greatest facilitation. This interval was considered equivalent to the time occupied by transmission of the force from the tendon to the muscle spindles, the initiation of impulses in the annulospiral endings, and impulse conduction in tibial nerve Ia fibres to the level of the knee. The stimulus intensities selected were just above threshold, that is, supraliminal, for the $\mathrm{T}$ and $\mathrm{H}$ reflexes. At the end of a sequence of 24 stimuli (see above) the stimulus strength was raised and the amplitude of the maximum $\mathbf{H}$ reflex was determined. The amplitude of the maximum tendon reflex was next obtained using a tendon hammer held in the hand, together with that of the response to the combined $\mathrm{H}$ and $\mathrm{T}$ stimuli. Four trials of each type of stimulation were averaged. Finally, the maximum evoked muscleo $\overrightarrow{0}$ response ( $M$ wave) after strong stimulation of the tibial nerve was measured.

RELAXED

WEAK CONTRACTION

(a)

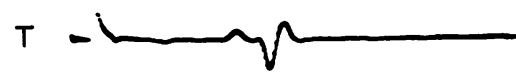

H

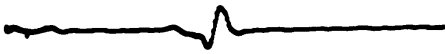

$\mathrm{HT}=$

M

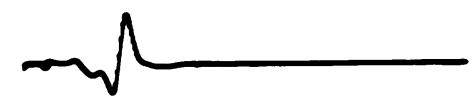

(b)

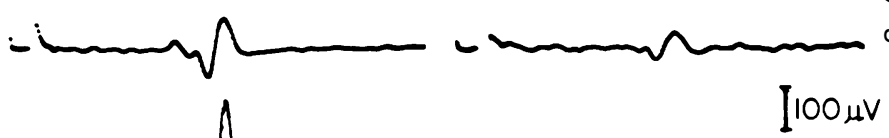

$110 \mathrm{mV}$ (c)
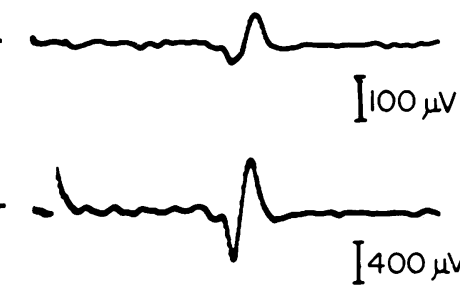

$$
\overrightarrow{\mathrm{Oms}}
$$

Fig. 3 Facilitation produced by a combination of $H$ and $T$ st:muli when the subject was relaxed (a) and making a weak voluntary contraction of the calf muscles (b and $c$ ). In $(b)$ the stimulus strength was the same as that in (a), while in (c) it was reduced so as to evoke $H$ and $T$ responses similar in size to those in $(a)$. The maximum evoked muscle response ( $M$ wave) is shown at the bottom. Note different amplitude calibrations. 


\section{Results}

A total of 41 trials were conducted on 15 subjects in the relaxed condition; in three of these subjects measurements were made on both legs. The observed amount of facilitation, $F$, varied markedly from one individual to another and also in the same subject depending on the level of arousal (see below). The greatest facilitation observed in any of the 41 trials was 17.6 and the mean value for the series was $4.26 \pm$ 3.78. When the values for the same leg were averaged the degree of facilitation ranged from 1.0 to 10.3 in different subjects. The experiment yielding the most facilitation in the resting state is shown in Fig. 3. The relatively small numbers of motoneurones participating in the supraliminal $H$ and $T$ reflexes can be gauged by comparison of these responses with that of the maximum evoked muscle response ( $M$ wave) at the bottom of Fig. 3 .

It was possible to demonstrate two experimental factors which influenced the degree of facilitation. These were the strength of the testing stimuli and the level of voluntary activation of the motoneurone pool.

\section{STIMULUS STRENGTH}

The effect of three stimulus intensities on the amount of facilitation is shown in Fig. 4. The intensities were supraliminal, maximal, and intermediate in relation to the sizes of the $\mathrm{T}$ and $\mathrm{H}$ reflexes. It can be seen that as the stimulus intensities increased, the single $T$ and $\mathrm{H}$ reflexes enlarged but the facilitation became less. When the facilitation was unity, the extra motoneurones recruited through spatial summation were exactly offset by those subjected to occlusion-that is, some neurones would have discharged to both the $\mathrm{T}$ and $\mathrm{H}$ reflex stimuli when the latter were presented separately. Inspection of Fig. 4 suggests that this condition would have been reached when the stimulus intensity was between the intermediate and maximal values. For occlusion to be total, the $\mathrm{T}$ and $\mathrm{H}$ reflexes must be equal in amplitude and no further increment in response should occur when the stimuli were presented together. The corresponding value for $F$ would be 0.5 . In the 15 experiments in which maximal stimuli were presented at rest full occlusion was achieved on only two occasions. In the remaining 13 experiments the maximum $T$ reflex was smaller than the maximum $\mathrm{H}$ reflex (Fig. 5). Interestingly, the responses to combined $\mathrm{H}$ and $\mathrm{T}$ stimulation $(H T$ responses) were significantly larger than the maximum $\mathrm{H}$ reflex on only one occasion and were at least $10 \%$ smaller than the latter in eight of 15 experiments. Possible reasons are given later (see Discussion). When the results were pooled (Fig. 4) the mean facilitation calculated for maximal stimulation was

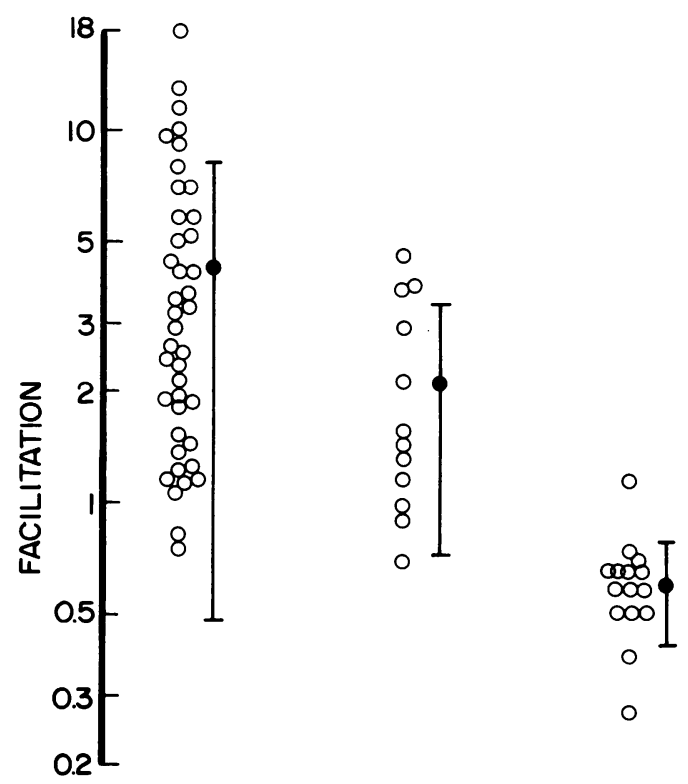

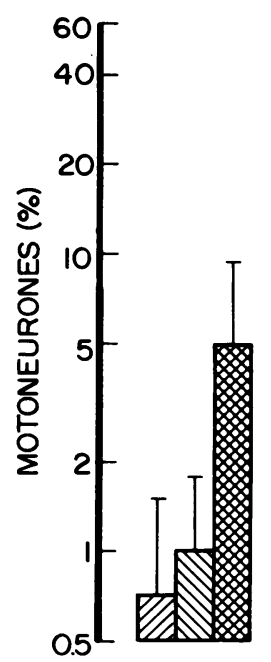

supraliminal

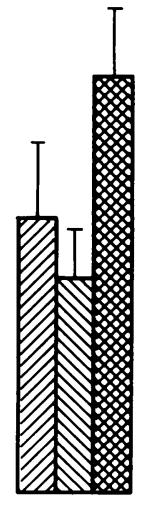

intermediate STIMULUS

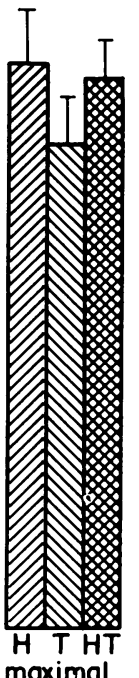

Fig. 4 Top. Facilitation (F) produced in relaxed subjects by stimulus intensities which were suprathreshold, intermediate, and maximal for corresponding tendon and $H$ reflexes. Mean values $\pm S D$ given at right of each group of results. Note logarithmic scale. Bottom. Mean $(+S D)$ reflex response amplitude to same three stimulus intensities described above. Results expressed on log scale as percentage of motoneurone pool discharging (using $M$ wave as index of motoneurone pool size). 


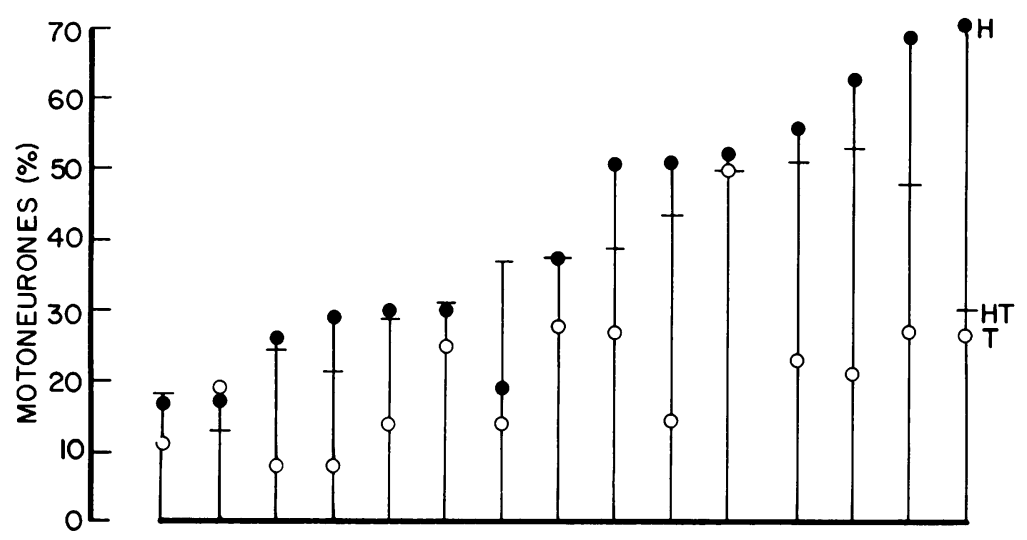

Fig. 5 Maximal $H$ and $T$ reflexes (filled and open circles respectively) in 12 subjects (15 legs), arranged in ranking order. The responses to paired $H$ and $T$ stimuli $(H T)$ are shown by horizontal bars. Results expressed as percentage of motoneurone pool discharging (using $M$ wave as index of motoneurone pool size).

0.6, ignoring those occasions when the $H T$ response was less than the $\mathrm{H}$ reflex.

\section{VOLUNTARY ACTIVATION}

The second experimental factor which could be shown to influence the amount of facilitation was voluntary activation of the motoneurone pool. If the supraliminal $\mathbf{H}$ and $\mathrm{T}$ stimuli were kept constant, the transition from rest to weak contraction of the calf muscles potentiated both reflexes in accord with previous observations (see, for example, Fig. 4 of Upton et al., 1971). The degree of facilitation, however, declined in all 14 pairs of observations. It could be argued that this reduction was simply a reflection of greater occlusion due to the increased populations of motoneurones involved in the $\mathrm{H}$ and $T$ reflexes, in keeping with the observations on the effects of increased stimulus strength (above). To test this possibility, further experiments were undertaken in which the stimulus intensity was reduced so that, during voluntary activity, the $\mathrm{H}$ and $\mathrm{T}$ reflexes were approximately of the same amplitude as those recorded at rest for slightly stronger stimuli. Even

Table Reflex responses and degrees of facilitation $(F)$ after stimulation during relaxation and weak contraction of calf muscles. The stimulus was suprathreshold at rest, was unchanged during contraction (middle column of results), and then reduced to be just above threshold again (right-hand column). The response amplitudes have been expressed as percentages of the maximum $M$ wave. Abbreviations as given in text

\begin{tabular}{llll}
\hline \multirow{2}{*}{ Stimulus } & \multicolumn{2}{l}{ Rest } & \multicolumn{2}{l}{ Weak contraction } \\
\cline { 2 - 4 } & Suprathreshold $(a)$ & As for $(a)$ & Reduced \\
\hline H & $0.71 \pm 0.76$ & $1.75 \pm 1.60$ & $0.91 \pm 0.96$ \\
T & $0.99 \pm 0.82$ & $2.54 \pm 1.54$ & $1.18 \pm 0.69$ \\
HT & $4.97 \pm 4.39$ & $6.12 \pm 3.79$ & $3.58 \pm 2.22$ \\
F & $4.3 \pm 3.8$ & $1.62 \pm 0.70$ & $1.85 \pm 0.84$ \\
Number & 41 & 14 & 19 \\
\hline
\end{tabular}

under these circumstances it was found that the degree of facilitation was substantially smaller than that observed at rest (Table).

\section{CRITICAL NUMBER OF EPSPS}

Using the curves in Fig. 2, an attempt was made to determine the critical numbers of EPSPs $(y)$ required to discharge soleus motoneurones innervating 187 legs of 15 subjects studied at rest. In three subjects (four legs) the calculated degree of facilitation was@ less than 2 and fell beyond the lower limits of the $\stackrel{\mathbb{Q}}{\circ}$ curves plotted in Fig. 2. The interpretation of thesec results was that the critical number of EPSPs in such subjects ranged from one to two. Unfortunately the mathematical analysis described in the introduction cannot be applied to such a situation and these experiments were, therefore, omitted from further consideration. For the remaining 12 subjects $(28$ experiments on 14 legs) the mean value for the critical number of EPSPs was $2.61 \pm 0.69$, the highest individual value being 4.4. As discussed below, these results are only applicable to the most excitable cells in each of the motoneurone pools and a special treatment is necessary to derive mean values for the full populations of cells.

\section{DIVERGENCE FACTOR}

The number of motoneurones supplied by a single Ia fibre was termed the divergence factor, $d$ (see Introduction). This factor was derived from Fig. 2 using the curve(s) appropriate to the sizes of the reflex responses and the observed degree of facilitation, $F$. In the 28 experiments which were suitable for this analysis (see above) the divergence values ranged from 64 to 304, the mean value being $139 \pm 62.3$.

\section{Discussion}

Although there have been other studies of synaptic responses resulting from inputs in two different Ia 
afferent nerve fibre populations, these have been concerned with the time course of changes in synaptic excitability (for example, Katz et al., 1977). The present study appears to be the first to give a detailed analysis of the phenomena of subliminal fringes and spatial summation. It has been possible to show that the degree of facilitation resulting from spatial summation varies several-fold among individuals and it also does so in any one subject on different occasions. Thus, the largest facilitation observed was 17.6 but in two subjects virtually none could be seen. In any one individual the change in facilitation with stimulus strength was that to be expected on the basis of Sherrington's classical studies (Sherrington, 1929). Thus, as the $\mathrm{H}$ and $\mathrm{T}$ stimuli became larger there was an increasing likelihood that some Ia fibres would be excited by both stimuli. This in turn would cause the same motoneurones to respond to the two types of stimulus. Under these circumstances the combined $(H T)$ response would be less than the sum of the $\mathrm{H}$ and $T$ reflexes. This type of behaviour was termed "occlusion" by Sherrington. As shown in the Results section, $\mathrm{H}$ and $\mathrm{T}$ stimulus intensities can be chosen so that the facilitation due to spatial summation is balanced by the diminution of response caused by occlusion. An additional finding was that the maximum tendon reflex potential was usually considerably smaller than that of the maximum $\mathrm{H}$ reflex. It is possible, though unlikely, that strong percussion of the Achilles tendon failed to excite all the spindle primary endings in the muscle. Some of the relative diminution of the $\mathrm{T}$ reflex may have been caused by dispersion in the arrival of impulses in Ia fibres at the cord. This asynchrony would be caused by the different times taken for the percussion wave to reach the muscle spindles and by the different lengths of Ia fibres. Both factors would, of course, depend on the positions of the muscle spindles within the muscle belly. A third factor causing a reduction in the size of $T$ reflex relative to the $H$ reflex could have been inhibition produced by the $T$ stimulus. This would account for the often smaller size of the $H T$ response compared with the $\mathrm{H}$ reflex. There was insufficient time between the $\mathrm{T}$ and $\mathrm{H}$ impulse volleys for this inhibition to be of the presynaptic type. A better explanation would be that the strong mechanical percussion had excited Golgi tendon afferent nerve fibres which would, in turn, cause disynaptic inhibition of homonymous motoneurones (Eccles et al., 1957).

The degree of facilitation was also found to depend on the background excitability of the motoneurone pool, being greater when the subject was relaxed than when a weak contraction was performed. This result was in keeping with theoretical predictions, for the threshold for reflex discharge would be less if the motoneurone excitability was raised-that is, since less motoneurone depolarisation would be required for impulse initiation, fewer EPSPs would be needed. In the introduction, it was shown that as $y$, the critical number of EPSPs, fell the subliminal fringe became smaller and the opportunity for spatial summation, and hence for facilitation, became less.

The critical number of EPSPs, $y$, required for impulse initiation ranged from less than 2 to 4.4 . However, this calculation depends on the assumption that all the cells in the motoneurone pool had similar thresholds, as depicted in Fig. 6 (left). This assumption is obviously false, for even with maximal stimulation of Ia fibres only about half the motoneurones responded with a reflex discharge (Fig. 5). The $y$ value should instead be regarded as an approximate mean value for the subgroup of neurones contributing to the supraliminal $\mathrm{H}$ and $\mathrm{T}$ reflexes (less than $2 \%$ of the total cell population). This subgroup is indicated by the lower arrow on the ordinate of Fig. 6 (right). In the latter graph the thresholds for the entire motoneurone pool are assumed to have a normal distribution about the mean so as to yield a cumulative frequency curve with a characteristic $S$ shape. The distance between the two arrows on the ordinate of Fig. 6 (right) represents the extra number of neurones recruited through spatial summation. It can be seen that the thresholds for these additional neurones will be higher than those for the cells giving the supraliminal $\mathrm{H}$ and $\mathrm{T}$ reflexes. Clearly, the most meaningful expression of motoneurone excitability would be the number of EPSPs required to excite cells

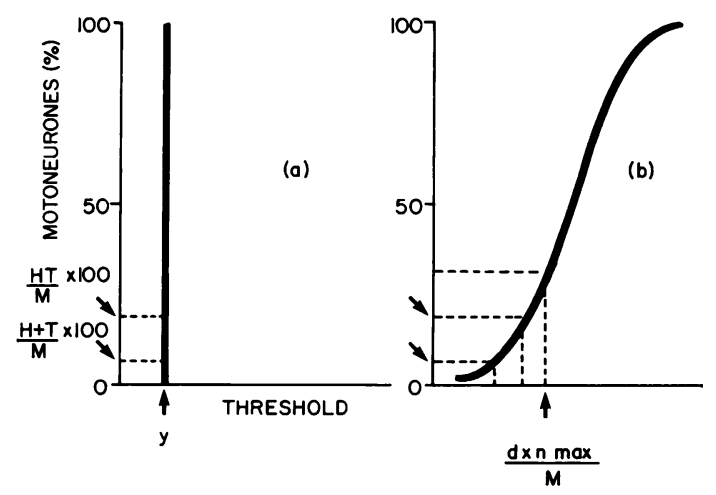

Fig. 6 Cumulative frequency plots of motoneurone thresholds, assuming that these are either identical (left) or distributed normally about the mean (right). The arrows on each ordinate indicate the neurones responding to the suprathreshold $H$ and $T$ stimuli given separately (lower arrow) or together (upper arrow). The top horizontal line intersecting the curve at right represents the cells responding to maximal reflex stimulation (see text). 
in the 50th percentile for a given individual. Computation of the $\mathbf{S}$ curve for such an individual would depend on having a second pair of co-ordinates to complement the pair already used for the low threshold segment of the curve. Provided two further assumptions are made, the second pair of co-ordinates can be determined by estimating the number of motoneurones discharging when all the Ia fibres have been excited. It is reasonable to assume that this condition is met when the maximum $\mathrm{H}$ reflex has been elicited. Certainly, adding a mechanical stimulus excites no additional motoneurones and therefore, presumably, no extra Ia fibres (see Results). The total number of EPSPs generated in the motoneurone pool after such maximal stimulation would be equal to the product of the total number of Ia fibres to the muscle and the divergence factor, $d$. The average number of EPSPs received by single motoneurones can be found by dividing this product by the number of cells in the motoneurone pool. Although the mean number of soleus motoneurones has been estimated in normal subjects as $957 \pm 254$ (McComas, 1977), there is no corresponding figure for the Ia fibres, or alternatively for the number of spindles, since each of the latter has a single Ia nerve fibre (Matthews 1972; Kennedy et al., 1975). Some guidance on this point may, however, be obtained from the anatomical study by Cooper (1966) and the review by Matthews (1972) in both of which numbers of a-motor fibres were compared with spindle populations for various mammalian muscles. Although the human soleus was not one of the muscles examined it is probable that the $a$-motor: spindle ratio would be about half that for the medial gastrocnemius, in keeping with observations in the cat and rat. Since the ratio for the medial gastrocnemius is about 5.5:1 in man (see Fig. 2 of Cooper, 1966), a reasonable estimate for the soleus might be $3: 1$, similar to the value for this muscle in the cat. If the $3: 1$ ratio is correct, the human soleus would have about 320 spindles, the same number as in the biceps brachii (see Table 1.1 of Matthews, 1972).

Using the estimated divergence value of 139 motoneurones per Ia nerve fibre (see Results) and an $\alpha$-motor: spindle ratio of $3: 1$, the average number of EPSPs received by a single motoneurone after maximal Ia activation would be $139 \times 1 / 3=46$. This degree of convergence was capable of exciting $17-71 \%$ of the motoneurone pools in the subjects studied at rest. The corresponding thresholds for the 50th percentile ranged from 38 to 103 EPSPs, the mean value being $62 \pm 20$.

The assumption that approximately 320 Ia fibres arise from muscle spindles in the soleus also permits calculation of the likelihood of any one fibre being involved in both the weak excitatory volleys employed to elicit the $\mathrm{H}$ and $\mathrm{T}$ reflexes respectively. Since the
Ia: a-MOTONEURONE SYNAPSE (HUMAN SOLEUS)

41 experiments on 18 legs

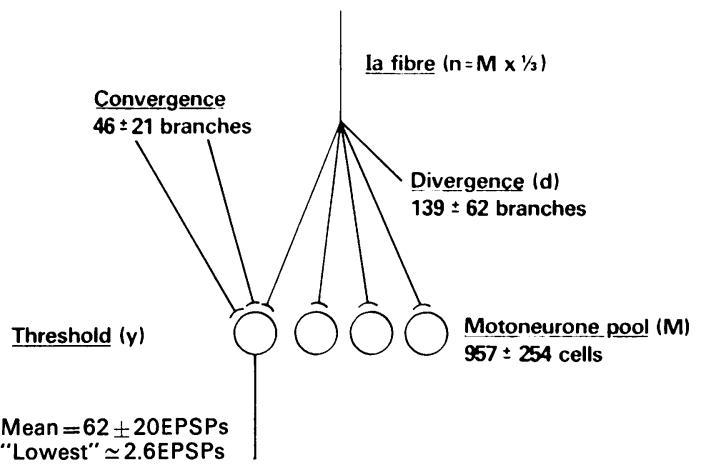

Fig. 7 Summary of mean estima:es for thresholds, convergence, and divergence (see text).

facilitation obtained in this investigation can be accounted for on the basis of volleys comprising not more than five Ia fibres (Fig. 2), the chance of any of five fibres activated by the $\mathrm{H}$ stimulus also being excited by the $\mathrm{T}$ stimulus (or vice versa) will be $5 \times 5 / 320=0.08$ (fibre), and therefore extremely unlikely.

How well do the present values, summarised in Fig. 7, compare with those obtained by direct measurement in animal experiments? In relation to divergence Mendell and Henneman (1971) found that a single Ia fibre would supply nearly all ( $93 \%$ ) of the 300 motoneurones in the medial gastrocnemius pool. In the cat semitendinosus Nelson and Mendell (1978) found a projection frequency of $82 \%$ for homonymous Ia fibres, the motoneurone population being 255 cells (Boyd and Davey, 1968). The present estimate of divergence, about 139 soleus motoneurones per Ia fibre, is rather lower than the cat values in absolute terms and, because of the larger motoneurone pool in man, it corresponds to a much smaller fraction of the latter. The excitability values are also somewhat smaller than those derived from animal experiments. Thus, a depolarisation of some $10 \mathrm{mV}$ is required to bring a motoneurone to the firing level (Eccles, 1957). The amplitude of a homonymous EPSP is about $100 \mu \mathrm{V}$, the most reliable values being derived from spike-triggered averaging and comprising $67 \mu \mathrm{V}$ (Watt et al., 1976), $91 \mu \mathrm{V}$ (Nelson and Mendell, 1978), $102 \mu \mathrm{V}$ (Mendell and Henneman, 1971), and less than $137 \mu \mathrm{V}$ (Mendell and Weiner, 1976). If the EPSPs after Ia activation were synchronous, approximately 100 would be needed to effect the $10 \mathrm{mV}$ depolarisation required to bring the motoneurone to the firing level. Although our present estimate of 62 EPSPs is considerably smaller than the above value it would not be surprising if the level of motoneurone excitability 
was appreciably lower in the animal experiments as a consequence of the anaesthesia and surgery employed. Alternatively, part of the discrepancy could be the result of underestimation of the extents of the subliminal fringes in the present experiments, because of inhomogeneity of thresholds even within the very small populations of neurones sampled. Despite the assumptions inherent in the present study, the method employed appears to offer a valid approach to the examination of motoneurone excitability in man. Increasing experience of the method, the introduction of technical refinements, and the acquisition of further information concerning the properties of the reflex loop, may all be expected to improve the reliability of the estimates in the future.

We are indebted to Dr Jean Delbeke for reviewing the manuscript and for correcting the mathematical treatment of the results. Dr Alan J. McComas received financial support from the Nuffield Foundation, Medical Research Council of Canada and the Muscular Dystrophy Association of Canada. He was on leave from the Department of Medicine (Neurology), McMaster University, Hamilton, Ontario, Canada during the course of the study. Dr Michael Mirsky's work was sponsored by the Alexander von Humboldt Foundation (Germany), and he was on leave from the Department of Neurology, Hadassah University Hospital, Jerusalem.

\section{References}

Boyd, I. A., and Davey, Mary R. (1968). Composition of Peripheral Nerves, p. 57. Livingstone: Edinburgh.

Burke, R. E. (1967). Composite nature of the monosynaptic excitatory postsynaptic potential. Journal of Neurophysiology, 30, 1114-1137.

Cooper, Sybil. (1966). Muscle spindles and motor units. In Control and Innervation of Skeletal Muscle, pp. 9-15. Edited by B. L. Andrew. Livingstone: Edinburgh.

Eccles, J. C. (1957). The Physiology of Nerve Cells, p. 270. Johns Hopkins Press: Baltimore.

Eccles, J. C., Eccles, R. M., and Lundberg, A. (1957). Synaptic actions on motoneurones caused by impulses in Golgi tendon organ afferents. Journal of Physiology, 138, 227-252.

Hoffmann, P. (1918). Über die Beziehungen der Sehnenreflexe zur willkürlichen Bewegung und zum Tonus. Zeitschrift für Biologie, 68, 351-370.
Katz, R., Morin, C., Pierrot-Deseilligny, E., and Hibino R. (1977). Conditioning of $\mathrm{H}$ reflex by a preceding subthreshold tendon reflex stimulus. Journal of Neurology, Neurosurgery, and Psychiatry, 40, 575-580.

Kennedy, W. R., Webster, H. de F., and Yoon, K. S. (1975). Human muscle spindles: fine structure of the primary sensory ending. Journal of Neurocytology, 4, 675-695.

Lundberg, A., and Winsbury, G. (1960). Selective activation of large afferents from muscle spindles and Golgi tendon organs. Acta Physiologica Scandinavica, 49, 155-164.

Magladery, J. W., Porter, W. E., Park, A. M., and Teasdall, R. D. (1951). Electrophysiological studies of nerve and reflex activity in normal man. IV. The two neurone reflex and identification of certain action potentials from spinal roots and cord. Bulletin of the Johns Hopkins Hospital, 88, 499-519.

Matthews, P. B. C. (1972). Mammalian Muscle Receptors and their Central Actions, p. 630. Edward Arnold: London.

McComas, A. J. (1977). Neuromuscular Function and Disorders, p. 364. Butterworths: London.

McComas, A. J., Mirsky, M., Struppler, A., and Velho, F. (1979). Indirect estimation of e.p.s.p.s. in human soleus motoneurones. Journa! of Physiology, 287, 14-15 P.

Mendell, L. M., and Henneman, E. (1971). Terminals of single Ia fibres: location, density and distribution within a pool of 300 homonymous motoneurones. Journal of Neurophysiology, 34, 171-187.

Mendell, L. M., and Weiner, R. (1976). Analysis of pairs of individual Ia-EPSPs in single motoneurones. Journal of Physiology, 255, 81-104.

Nelson, S. G., and Mendell, L. M. (1978). Projection of single knee flexor Ia fibers to homonymous and heteronymous motoneurons. Journal of Neurophysiology, 41, 778-787.

Sherrington, C. S. (1929). Some functional problems attaching to convergence. Preceedings of the Royal Society, B, 105, 332-362.

Upton, A.R.M., McComas, A. J., and Sica, R.E.P. (1971). Potentiation of "late" responses evoked in muscles during effort. Journal of Neurology, Neurosurgery, and Psychiatry, 34, 699-711.

Watt, D. G. D., Stauffer, E. K., Taylor, A., Reinking, R. M., and Stuart, D. G. (1976). Analysis of muscle receptor connections by spike-triggered averaging. 1. Spindle primary and tendon organ afferents. Journal of Neurophysiology, 39, 1375-1392. 